\title{
To explain Crab pulsar emission by ICS Model
}

\author{
J.L. Han \\ Beijing Astronomical Observatory, CAS, Beijing 100080, China \\ B. Zhang, G.J. Qiao, R.X. Xu \\ Department of Geophysics, Peking University, Beijing 100871, China
}

\begin{abstract}
Primary results of our recent work show that radio, infrared and optical, X-ray and $\gamma$-ray emission of Crab pulsar might be explained by Inverse Compton Scattering (ICS) mechanism.
\end{abstract}

Crab pulsar is mysterious since it emits not only radio pulses, but the pulses at the infrared, optical, X-ray and gamma-ray bands as well. How normal pulsars emit radio pulses is a long-standing theoretical work to be done. The similar pulse shapes of Crab pulsar, especially the emission bridge (see Moffett \& Hankins 1996), at radio to infrared, optical, X-ray and gamma-ray bands, tell us that all those radiation is produced by the somewhat the same process from single magnetic pole, though probably from different emission regions.

Recently, ICS process has been proved to be the most important process for relativistic particles to loss their energy near the surface of the neutron stars (cf. Sturner 1995; Dermer 1990; Zhang \& Qiao 1995; Qiao \& Zhang 1996). According to our primary simulation results, we understand that the emission of Crab pulsar at every band might be produced by the same process - ICS mechanism.

(1). Radio emission is explained as being from the ICS process of the secondary outflowing particles with the low frequency wave produced by the inner gap sparking. That is to say,

$$
\omega_{\text {sparking }}+\mathrm{e}_{\text {secondary }}^{+,-} \Longrightarrow \text { observed radio emission }
$$

It has been shown by Qiao (1988) that ICS process is more efficient to produce radio emission than the curvature radiation. The spectral index of observed radio emission $\alpha=3.1$ can be reproduced in our simulation by angular and non resonant scattering, after taking the index of electron energy spectrum $p=0.6$.

As the spectral index of relativistic particles becomes flatter as they flow out, because those particles with larger Lorentz factor would loss more energy due to ICS process with thermal photons (producing X-ray emission), we can therefore easily explain why core emission that is generated nearer to neutron star surface in ICS model has a steeper spectrum while conal emission from higher altitudes has a flatter one.

(2). The spectrum of infrared and optical pulse emission could not be fitted by a black-body spectrum (Middleditch et al. 1983). We understand that they are produced by the ICS process of secondary particles with thermal photons $\epsilon_{\text {th }}$ 
of about a low $10^{3} \mathrm{~K}$, perhaps from angular dependent temperature component (Zavlin et al. 1995) from the whole neutron star. That is to say,

$$
\epsilon_{\text {th,low }}+\mathrm{e}_{\text {secondary }}^{+,-} \Longrightarrow \text { observed infrared and optical emission }
$$

We find from our simulation that the infrared and optical pulses, if they are produced by ICS process, must be generated in strong magnetic fields $(\log B>11.5)$. Otherwise, there should have an excess emission at X-ray band.

(3). The observed (soft) X-ray spectrum is produced by ICS process of the outgoing primary positrons and secondary particles with the thermal photons $\epsilon_{\mathrm{th}}$ of the high temperature from the (heated polar cap of) neutron star, so that pulsar X-ray emission is closely related to the temperature of the neutron stars. Only young pulsars (cf. Urpin \& Shalybkov 1995) or accretion pulsars possibly have enough high temperature (around the polar cap), and hence can emit X-ray pulses with ICS process. The process then is

$$
\epsilon_{\text {th,high }}+\left(\mathrm{e}_{\text {primary }}^{+} \& \mathrm{e}_{\text {secondary }}^{+,-}\right) \Longrightarrow \text { observed outward X-ray emission }
$$

(4). The $\gamma$-ray emission is produced by the ICS process of primary electrons with inwards going X-ray photons, where these X-ray photons come from the ICS of the cascade inward-going electrons with the thermal photons from the surface of neutron star and has a power-low spectrum. That is

$$
\begin{gathered}
\epsilon_{\text {th,high }}+\mathrm{e}_{\text {primary }}^{-} \Longrightarrow \mathrm{X}-\text { ray }_{\text {inwards }} \\
\mathrm{X}-\text { ray }_{\text {inwards }}+\mathrm{e}_{\text {secondary \& primary }}^{-} \Longrightarrow \text { observed gamma-ray }
\end{gathered}
$$

Sturner \& Dermer (1994) have fitted the gamma-ray spectrum of Crab pulsar by the ICS process of the monoenergetic electrons with the observed power-law $\mathrm{X}$-ray photons. We emphasize here that the X-ray photons for such a upperscattering are not the observed outgoing $\mathrm{X}$-ray photons, but the inward-going photons.

Acknowledgments. We thank National Nature Science Foundation of China. for financial support. JLH also acknowledges the supports from Science Foundation for Young Researchers of CAS and the Director Foundation of BAO.

\section{References}

Dermer C., 1990, ApJ 360, 197

Moffett D.A., Hankins T.H., 1996, ApJ, in press

Middleditch J., Pennypacker C., Burns M.S., 1983, ApJ 273, 261

Qiao G.J., 1988, in: High Energy Astrophysics, Bonner G.B. (ed), SpringerVerlag, p.88

Qiao G.J., Zhang B., 1996, A\&A 306, L5

Sturner S.J., 1995, ApJ 446, 292

Urpin V.A., Shalybkov D.A., 1995, A\&A 294, 117

Zavlin V.E., Pavlov G.G., Shibanov Yu.A., Ventura J., 1995, A\&A 297, 441

Zhang B., Qiao G.J., 1995, A\&A, in press 